Vietnam Journal of Mechanics, VAST, Vol.37, No. 2 (2015), pp. 91 -104

DOI:10.15625/0866-7136/37/2/5144

\title{
ACTUATOR SATURATION CONTROL OF A BENCHMARK STRUCTURE USING HEDGE-ALGEBRAS-BASED FUZZY CONTROLLER
}

\author{
Bui Hai Le \\ Hanoi University of Science and Technology, Hanoi, Vietnam \\ E-mail: le.buihai@hust.edu.vn \\ Received October 29, 2014
}

\begin{abstract}
In this paper, the problem of actuator saturation control of a benchmark structure using hedge-algebras-based fuzzy controller $(\mathrm{HAC})$ is presented. In HAC, linguistic values of linguistic terms are obtained through semantically quantifying mappings (SQMs) based on few fuzziness parameters of each linguistic variable without using any fuzzy set and inherent order relationships between linguistic values of each linguistic variable are always ensured. Hence, the design of a HAC leads to determining parameters of SQMs, which are fuzziness measures of primary terms and linguistic hedges occurring in a fuzzy model. As a case study, a HAC is designed to actuator saturation control of a benchmark structure with active bracing system (ABS) subjected to earthquake excitation. Control performance of the controller is also discussed in order to shown advantages of the proposed method.
\end{abstract}

Keywords: Structural active control, actuator saturation, hedge algebras, hedge-algebrasbased fuzzy controller, earthquake excitation.

\section{INTRODUCTION}

The reduction of response quantities such as velocities, deflections, and forces induced by environmental dynamic loadings (i.e., wind and earthquake) is very necessary in order to increase the strength and safety of structures. The reduction of undesired vibration of structures has become an attractive subject of research, and many structural control methods have been implemented in practice [1].

Fuzzy set theory introduced by Zadeh in 1965 has been widely applied in many real situations because it is a useful mathematical tool to model uncertain and vague data. Many researches on vibration control of structures using fuzzy controllers have been reported. Pourzeynali et al. [2] designed and optimized different parameters of an active tuned mass damper control scheme to get the best results in the reduction of building responses under earthquake excitations using genetic algorithms and fuzzy logic. In

(C) 2015 Vietnam Academy of Science and Technology 
the study conducted by Guclu and Yazici [3], fuzzy and proportional-derivative (PD) controllers have been designed for active control of a real building against earthquake. Marinaki et al. [4] proposed and tested the particle swarm optimization (PSO) for the calculation of the free parameters in active control of smart piezoelastic beams using fuzzy controller. Wanga et al. [5] presented a problem of active compensation control of friction-induced self-excited vibration of a mass on a moving belt system using adaptive fuzzy systems. Das et al. [6] presented a fuzzy-logic control algorithm, based on the fuzzification of the MR damper characteristics, for the semiactive control of building frames under seismic excitation. Uz et al. [7] proposed an optimal design strategy based on genetic algorithms (GA) using fuzzy controller for active control of a structure with nonlinear hysteretic control devices.

Although a fuzzy controller is flexible and easy to use, its semantic order of linguistic values is not closely guaranteed and its fuzzification and defuzzification methods are quite complicated [1].

Hedge algebras (HAs) theory, first introduced in 1990, see [8-15] and the references therein, showed that linguistic values can formulate an algebraic structure. It is a complete hedge algebra structure with the main property of which, semantic order of linguistic values, is always guaranteed. It is even a rich enough algebraic structure, and therefore, it can describe completely reasoning processes. HAs can be considered as a mathematical order-based structure of terms-domains, the ordering relation of which is induced by the meaning of linguistic terms in these domains. It is shown that each terms-domain has its own order relation called semantically ordering one induced by the meaning of the terms. Many interesting semantic properties of terms can be formulated using this relation, and some of these can be taken to form an axiom system of HAs. Application of hedge-algebras-based fuzzy controller (HAC) in structural active fuzzy control with remarkable results in [1,16-19] provided a new approach to study problems of vibration control of structures. However, the actuator saturation, a very important problem in the field of structural control because any actuation mechanisms are subject to inherent physical limitations, was still not considered in [1,16-19].

In this paper, the problem of actuator saturation control of a benchmark structure with active bracing system (ABS) subjected to earthquake excitation using hedgealgebras-based fuzzy controller (HAC) is presented to show advantages of the proposed controller.

\section{PROBLEM UNDER CONSIDERATION}

In this section, a benchmark three-storey shear-beam building model subjected to earthquake excitation $\ddot{x}_{0}$ shown in Fig. 1 studied by Du et al. in 2011 [20] with actuator saturation is considered for controller design.

The motion equation of the structure system with actuator saturation is expressed as

$$
[M]\{\ddot{x}\}+[C]\{\dot{x}\}+[K]\{x\}=\operatorname{sat}(u)-\{\delta\} \ddot{x}_{0},
$$

where $\{x\}=\left[\begin{array}{lll}x_{1} & x_{2} & x_{3}\end{array}\right]^{T},\{\delta\}=\left[\begin{array}{lll}m_{1} & m_{2} & m_{3}\end{array}\right]^{T}$. 


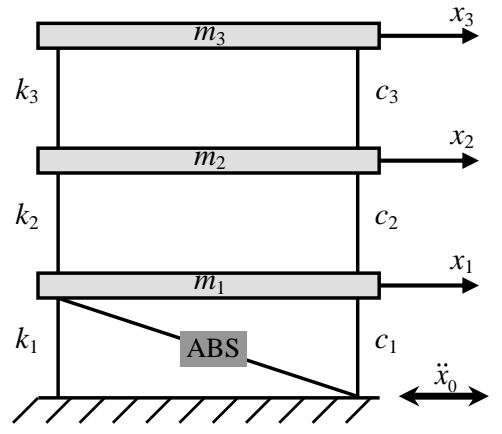

$m_{i}=1000 \mathrm{~kg}, c_{i}=1.407 \mathrm{kNs} / \mathrm{m}$, and $k_{i}=980 \mathrm{kN} / \mathrm{m}$, where $i=1,2,3$, respectively.

The ABS is installed at the first floor to control the vibration of the structure.

The 1940 El Centro earthquake of which peak ground acceleration is scaled to $0.112 \mathrm{~g}$ is used as excitation load $\ddot{x}_{0}$ of the structure.

Fig. 1. The structural system and parameters

Control force $u$ is generated by the ABS installed on the first floor and sat $(u)$ with limit of $u_{\lim }$ for the actuator is described as follows [20]

$$
\operatorname{sat}(u)=\left\{\begin{array}{ccc}
u_{\lim } & \text { if } & u \geq u_{\lim } \\
u & \text { if } & -u_{\lim } \leq u \leq u_{\lim } \\
-u_{\lim } & \text { if } & u \leq-u_{\lim }
\end{array}\right.
$$

The $n \times n(n=3)$ matrices $[M],[C]$ and $[K]$ represent the structural mass, damping and stiffness matrices, respectively.

The goal of the designed controller is to reduce dynamic responses in the first floor, and hence responses in the structure. Control diagram of the controller with two-input state variables $x_{1}$ and $\dot{x}_{1}$ and one-output control variable $u$ is shown in Fig. 2.

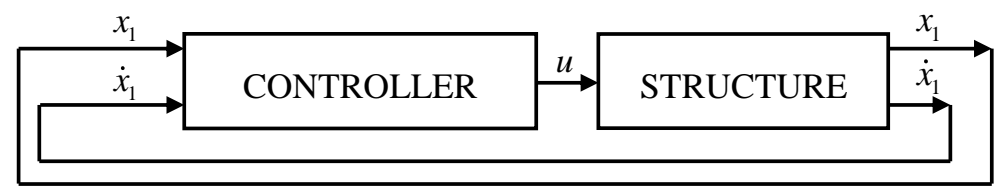

Fig. 2. Control diagram

It is assumed that the reference domains of the state variables and the control variable are given by $-a_{0} \leq x_{1} \leq a_{0},-b_{0} \leq \dot{x}_{1} \leq b_{0}$ and $-c_{0} \leq u \leq c_{0}$.

\section{CONTROLLER DESIGN}

The idea and basic formulas of HAs theory based on definitions, theorems, and propositions in [1,8-19] are summarized and presented in Appendix 1.

Consider a HAs structure $A X=(X, G, C, H, \leq)$, where $G=\{$ Negative, Positive $\}$ and $H=\{$ Very, Little $\}$. In this case, $p=q=1$, and as a result, SQM values $\varphi$ for all linguistic values of the term-set $X$ are determined through only two independent fuzziness parameters, which are $0<f m\left(c^{-}\right)$and $\mu\left(h^{-}\right)<1$. Some typical linguistic values with SQM values of the term-set $X$ are calculated and arranged in Tab. 1 for the case of $f m\left(c^{-}\right)$and 
$\mu\left(h^{-}\right)=0.5$. Where, Ne, Po, $V$ and $L$ stand for Negative, Positive, Very and Little, respectively.

Hence, by utilizing HAs theory to describe term-set of a linguistic variable, inherent order relationships between linguistic values existing in the term-set are closely guaranteed.

Table 1. Some typical linguistic values with SQM values

\begin{tabular}{|c|c|c|c|c|c|c|c|}
\hline Linguistic values & $V N e$ & $N e$ & $L N e$ & $W$ & $L P o$ & $P o$ & $V P o$ \\
\hline SMQ values $\varphi$ & 0.125 & 0.25 & 0.375 & 0.5 & 0.625 & 0.75 & 0.875 \\
\hline
\end{tabular}

Next, designing steps of the hedge-algebras-based fuzzy controller (HAC) for active control of the structure are presented. Operation principle of HAC with two-input state variables $x_{1}$ and $\dot{x}_{1}$ and one-output control variable $u$ is shown in Fig. 3 [19].

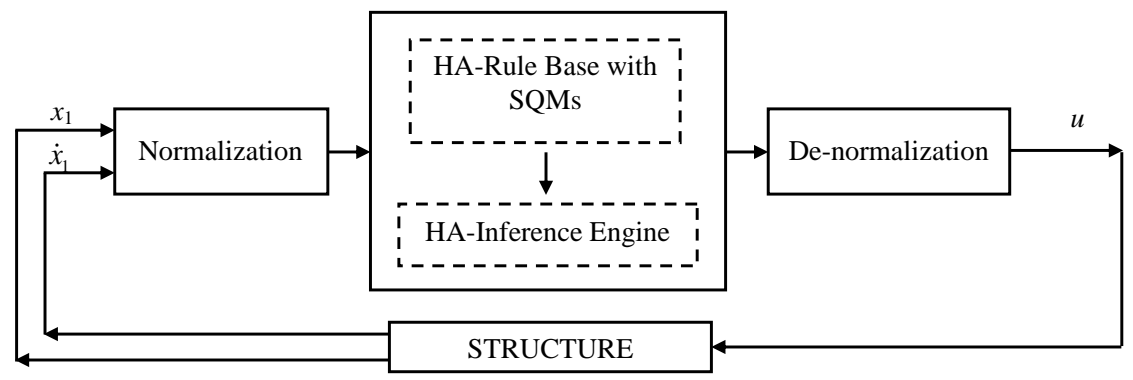

Fig. 3. Operation principle of HAC

Although linguistic variables $x_{1}, \dot{x}_{1}$ and $u$ under consideration are different, their hedge algebras are here defined with a similar HAs structure $A X$ as shown in above with the case of $f m\left(c^{-}\right)=0.5$ and $\mu\left(h^{-}\right)=0.5$. The components of the controller are presented as follows [19]:

- Normalization: the reference domains of the linguistic variables $X$, given in the form of an interval $[a, b]$, must be normalized into the domain of a SQM, given in $[0,1]$, by the unique transformation: $g_{X}:[a, b] \rightarrow[0,1]$. These mappings for state variables are presented in Fig. 4.
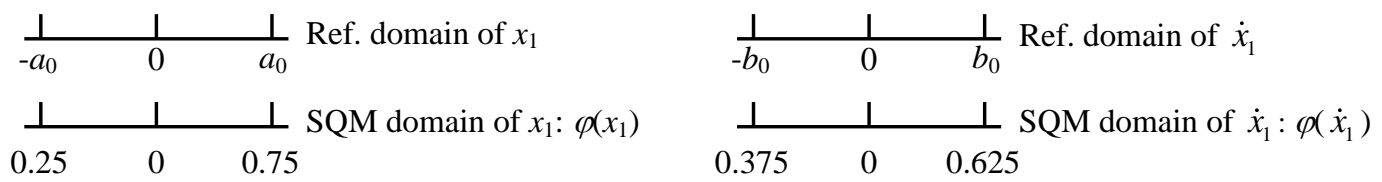

Fig. 4. Normalization of the state variables

- HA-Rule Base with SQMs: a typical fuzzy rule base stored in the form of if-then rules with SQM values is considered for HAC as shown in Tab. 2. 
Table 2. HAC rule base with SQM values

\begin{tabular}{|l|l|l|l|}
\hline$\varphi\left(x_{1}\right)$ & LNe: 0.375 & $W: 0.5$ & LPo: 0.625 \\
\hline Ne: 0.25 & VNe: 0.125 & Ne: 0.25 & LNe: 0.375 \\
\hline LNe: 0.375 & Ne: 0.25 & LNe: 0.375 & $\boldsymbol{W}: 0.5$ \\
\hline W: 0.5 & LNe: 0.375 & $\mathbf{W}: 0.5$ & LPo: 0.625 \\
\hline LPo: 0.625 & $\mathbf{W}: 0.5$ & LPo: 0.625 & Po: 0.75 \\
\hline Po: 0.75 & LPo: 0.625 & Po: 0.75 & VPo: 0.875 \\
\hline
\end{tabular}

- HA-Inference Engine: the semantically quantifying surface (SQS) established through the points that present the control rules occurring in Tab. 2 as shown in Fig. 5 are here considered as the HA inference engine to infer the control force $u$ for given HA input $\left(x_{1}\right.$ and $\left.\dot{x}_{1}\right)[1]$.

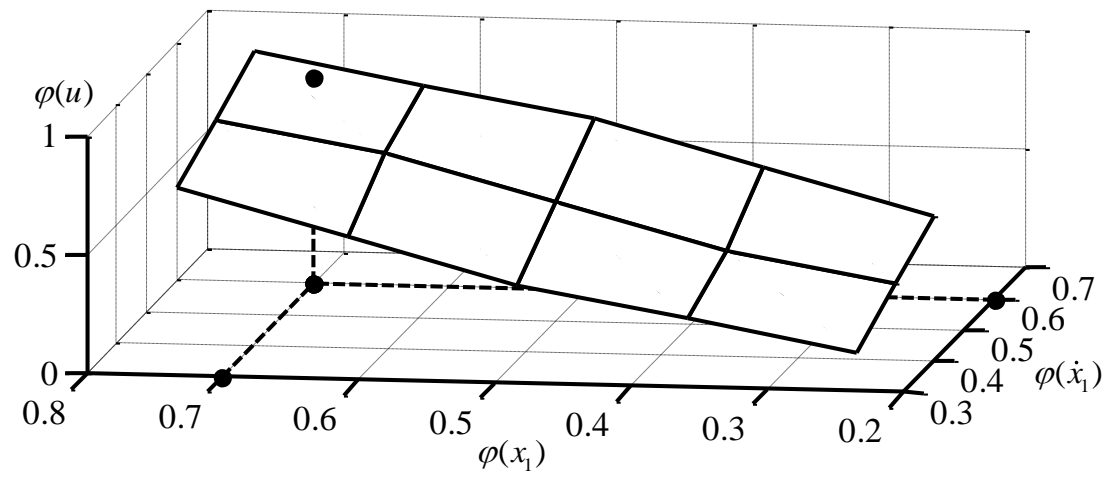

Fig. 5. HA-Inference Engine

- De-normalization: in order to convert from the SQM value to real one of the control variable $u$, the de-normalization step is needed and based on an opposite mapping $g_{X}^{-1}$ of $g_{X}$ as shown in Fig. 6.

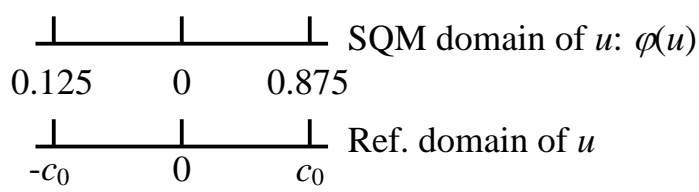

Fig. 6. De-normalization of the control variable

By regarding the designing steps of $\mathrm{HAC}$, some characteristics of the controller HAC for structural active control could be found as follows: 
- Their components and operation principle are similar to those of a conventional fuzzy controller (denoted by FC) for structural active control that contains two-input state variables $x_{1}$ and $\dot{x}_{1}$ and one-output control force $u$. This analogical FC is presented in Appendix 2.

- The inherent semantic order between the linguistic values based on their SQMs is closely guaranteed and expressed instead of using any fuzzy sets.

- The HA-Rule Base with SQMs could be clearly observed by utilizing the semantically quantifying surface (SQS).

- The normalization, HA-Inference Engine and de-normalization components are easily operated by using very simple linear interpolations.

- As a result, it can be emphasized that the hedge-algebras-based fuzzy controller are easy in establishment, coherent in implementation and efficient in computation time [19].

\section{NUMERICAL RESULTS}

In this section, two important criteria for building structures subjected to external dynamic loads are considered as follows:

- Peak storey drift:

$$
J_{1}=\max _{t, i}\left(\frac{\left|d_{i}(t)\right|}{d_{\max }}\right)
$$

- Peak absolute acceleration:

$$
J_{2}=\max _{t, i}\left(\frac{\left|\ddot{x}_{a i}(t)\right|}{\ddot{x}_{a \max }}\right)
$$

The criteria $J_{1}$ and $J_{2}$ respectively relate to structural safety and human tolerance. Where, $d_{i}(t)$ is the storey drift of the $i^{t h}$ floor in the controlled response, $d_{\text {max }}$ is the pick storey drift in the uncontrolled response, $\ddot{x}_{a i}(t)$ is the absolute acceleration of the $i^{t h}$ floor in the controlled response and $\ddot{x}_{a \max }$ is the pick absolute acceleration in the uncontrolled response.

First, the actuator limitation is given as $u_{\lim }=700 \mathrm{~N}$. The values of the criteria $J_{1}$ and $J_{2}$ for the structure are given in Tab. 3, where the results given from other methods are also included for comparison. It is found that the proposed controller provides quite good results in reducing the pick storey drift as well as pick absolute acceleration. The results obtained from the HAC are quite better than those of the analogical FC in term of both criteria $J_{1}$ and $J_{2}$.

Table 3. Normalized maximum values of responses in the case of $u_{\lim }=700 \mathrm{~N}$

\begin{tabular}{|c|c|c|c|c|c|c|c|}
\hline Controllers & LQR [21] & MBBC [21] & SSMC [21] & Lim et al. [21] & Du et al. [20] & FC & HAC \\
\hline$J_{1}$ & 0.657 & 0.381 & 0.388 & 0.396 & 0.41 & 0.491 & 0.460 \\
\hline$J_{2}$ & 0.584 & 0.548 & 0.560 & 0.543 & 0.53 & 0.587 & 0.551 \\
\hline
\end{tabular}

Time responses of the storey drift of the first floor, the absolute acceleration of the third floor and the control force in the case of $u_{\text {lim }}=700 \mathrm{~N}$ are shown in Figs. 7-9, 
respectively. It can be seen from Figs. 7-9 that a quite similar variation of the storey drift of the first floor, the absolute acceleration of the third floor and the control force of the controllers FC and HAC could be found for the case of $u_{\text {lim }}=700 \mathrm{~N}$.

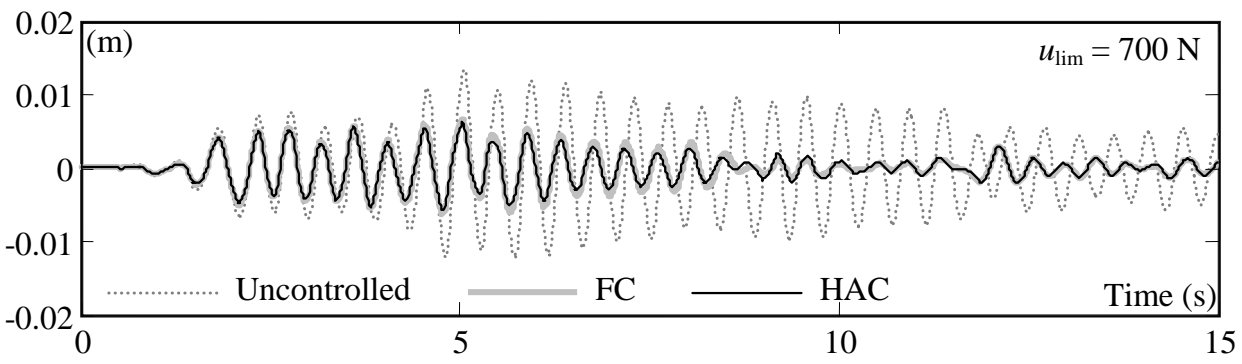

Fig. 7. Storey drift of the first floor in the case of $u_{\lim }=700 \mathrm{~N}$

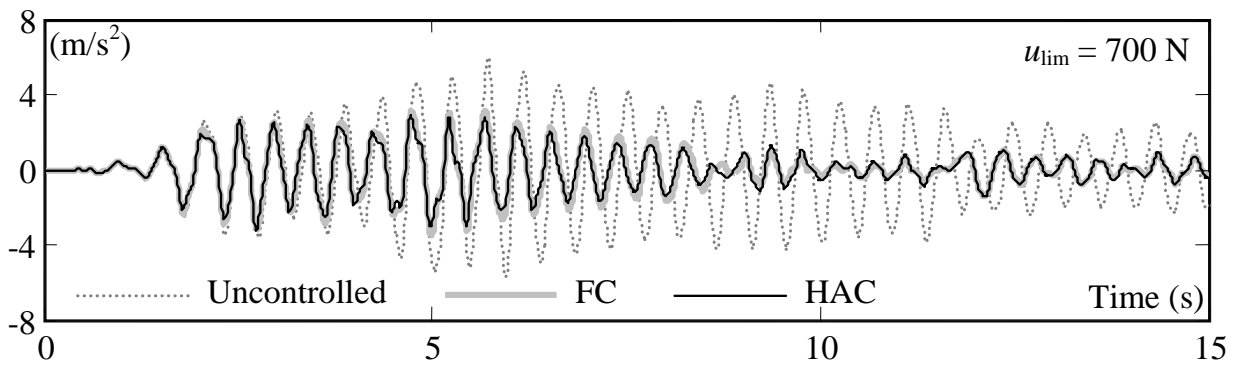

Fig. 8. Absolute acceleration of the third floor in the case of $u_{\lim }=700 \mathrm{~N}$

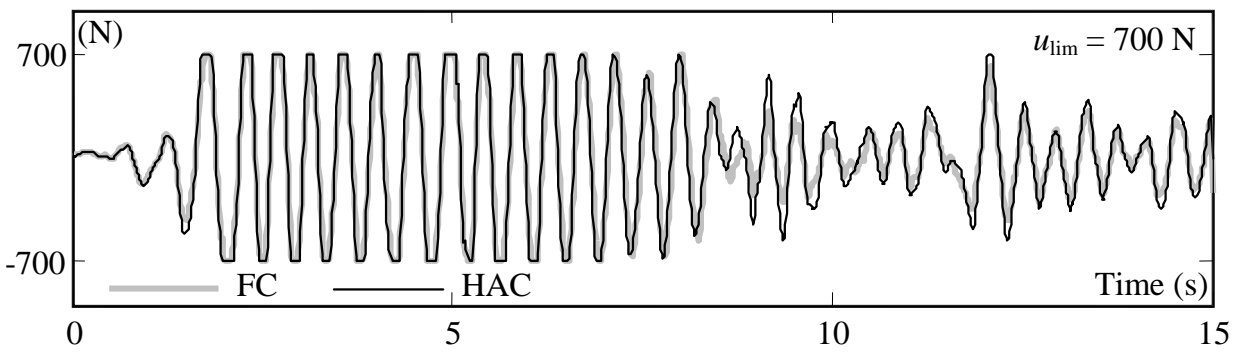

Fig. 9. Control force in the case of $u_{\lim }=700 \mathrm{~N}$

Next, in order to study the influence of the actuator limitation on the criteria $J_{1}$ and $J_{2}$, different values of $u_{\text {lim }}$ are considered and simulation results are plotted in Figs. 10-11. It can be found that the performance of HAC is quite better than that of FC for all cases of actuator limitations as well as for both criteria $J_{1}$ and $J_{2}$. 


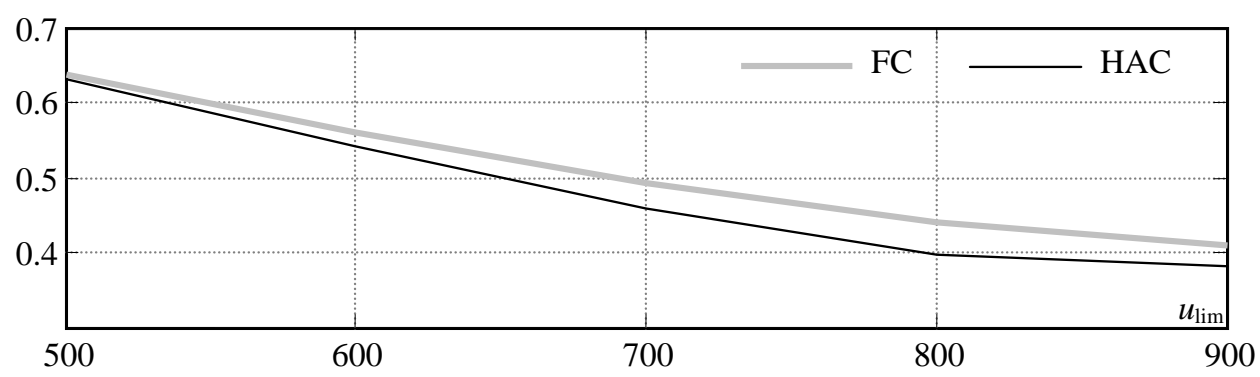

Fig. 10. Variations of peak storey drift versus actuator limitation

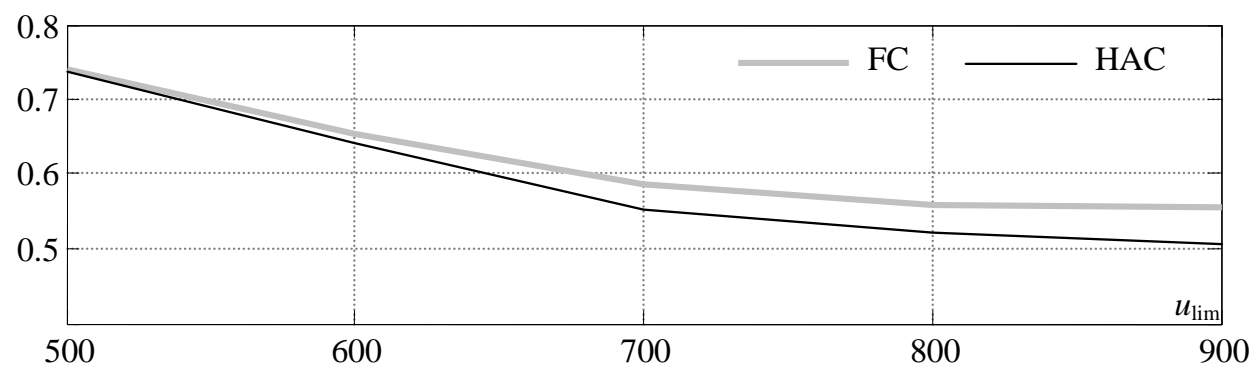

Fig. 11. Variations of peak absolute acceleration versus actuator limitation

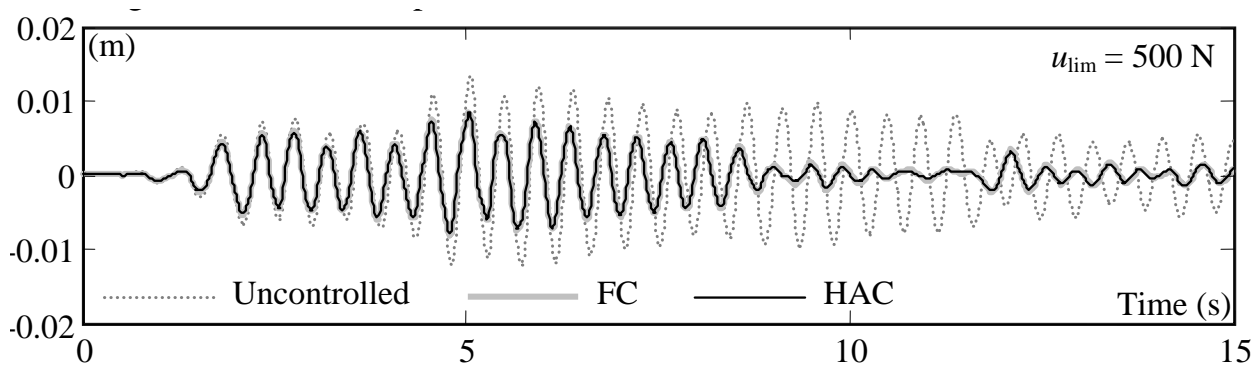

Fig. 12. Storey drift of the first floor in the case of $u_{\lim }=500 \mathrm{~N}$

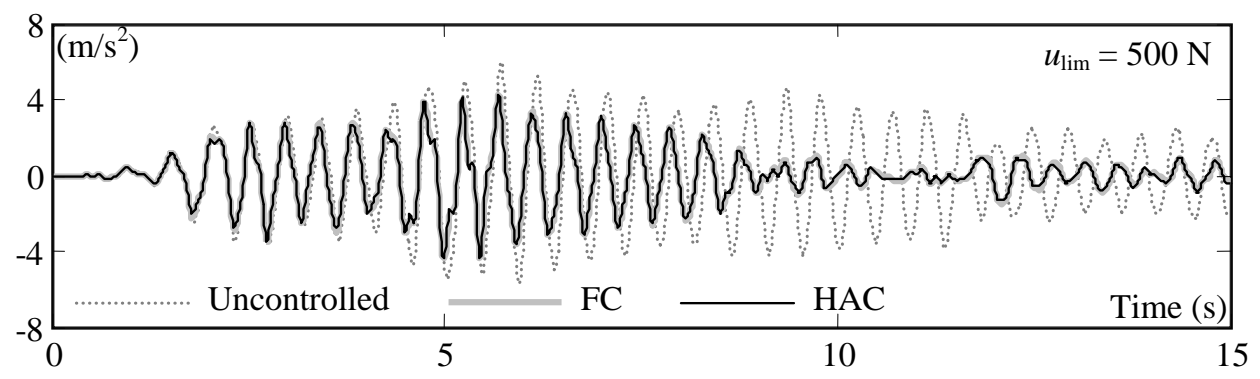

Fig. 13. Absolute acceleration of the third floor in the case of $u_{\lim }=500 \mathrm{~N}$ 


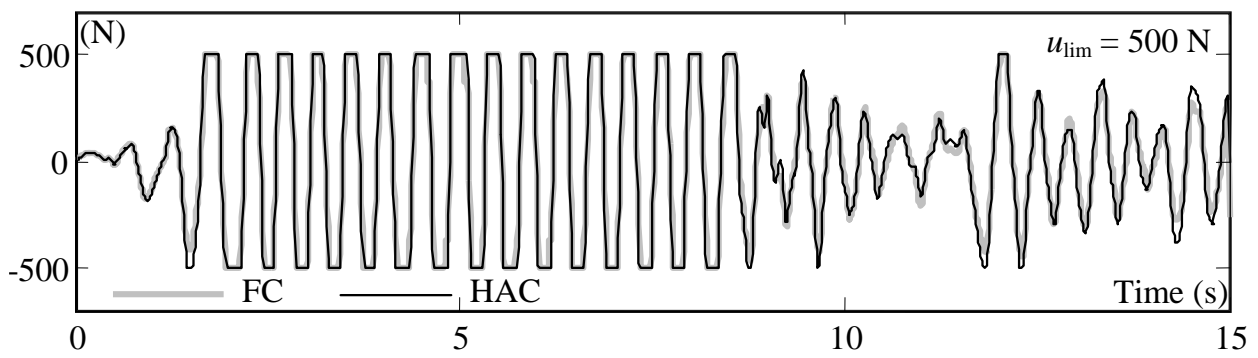

Fig. 14. Control force in the case of $u_{\lim }=500 \mathrm{~N}$

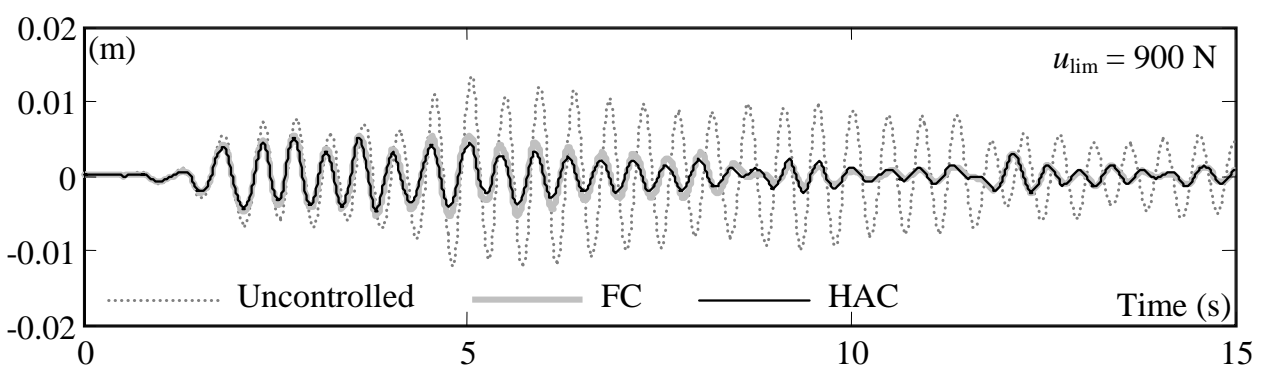

Fig. 15. Storey drift of the first floor in the case of $u_{\text {lim }}=900 \mathrm{~N}$

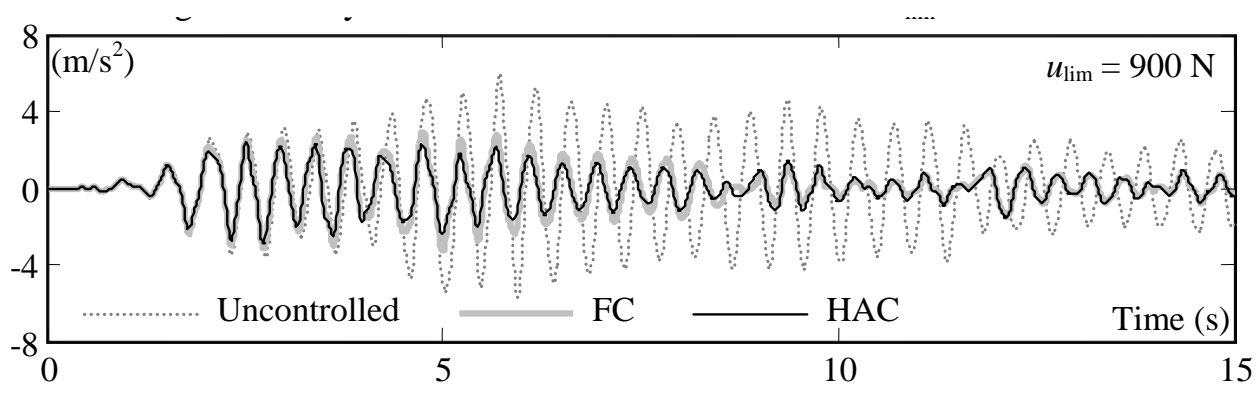

Fig. 16. Absolute acceleration of the third floor in the case of $u_{\lim }=900 \mathrm{~N}$

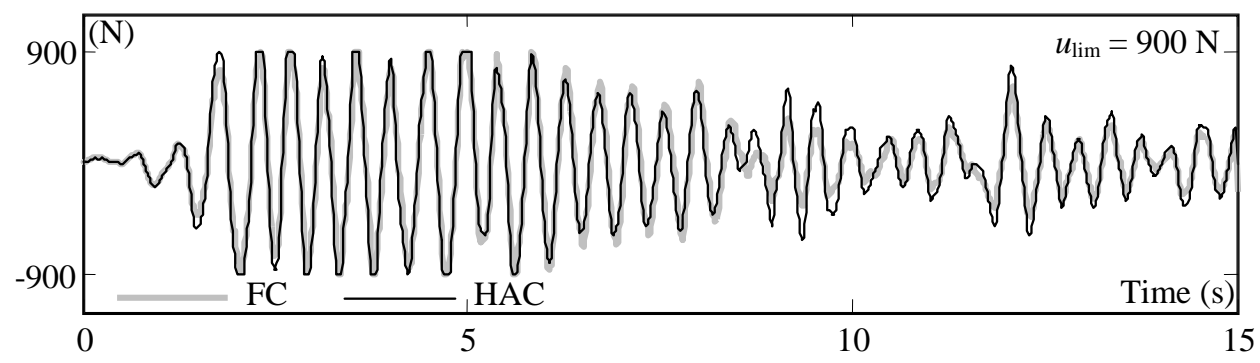

Fig. 17. Control force in the case of $u_{\lim }=900 \mathrm{~N}$ 
Time responses of the storey drift of the first floor, the absolute acceleration of the third floor and the control force in the cases of $u_{\text {lim }}=500$ and $900 \mathrm{~N}$ are plotted in Figs. $12-17$, respectively.

It can be observed in Figs. 12-17 that the responses of the storey drift, the absolute acceleration and the control force obtained from the controllers are also quite similar the cases of $u_{\text {lim }}=500 \mathrm{~N}$ and $u_{\text {lim }}=900 \mathrm{~N}$.

The results obtained from the HAC are quite better than those of the analogical FC in term of both criteria $J_{1}$ and $J_{2}$ for all cases of $u_{\text {lim }}$. However, the improvements are small, about $6 \%$. Both controllers, HAC and FC, are simulated base on similar control rule bases (see Tab. 2 and Tab. 5 in Appendix 2) and the same condition of the actuator saturation. Hence, above small differences could be due to the differences in components of the controllers HAC and FC: Normalization and Fuzzification, HA-Inference Engine and FC-Inference Engine and De-normalization and De-fuzzification.

Table 4. Comparison of CPU computation time (s) of fuzzy controllers

\begin{tabular}{|c|c|c|}
\hline Controller & FC & HAC \\
\hline Computation time & 13.42 & 1.09 \\
\hline Reduction ratio (\%) & 0 & 91.88 \\
\hline
\end{tabular}

In order to illustrate the computation performance of the proposed controller, computation times (CPU times) of HAC and FC are measured in the case of $u_{\text {lim }}=700 \mathrm{~N}$ and presented in Tab. 4. It is shown that the CPU time of HAC is much reduced by over $90 \%$ in comparison with that of the analogical FC. The CPU times are measured when running computer programs on the machine ASUS U46E with 8GB RAM, the OS is Windows 7 Home Premium, the programming language is Matlab 7.6.0, the total time of simulation is $15 \mathrm{~s}$ and the time step size is $0.01 \mathrm{~s}$. It is a significant benefit of HAC in practical control because computation time of control forces is one of the main factors of input time delay of a controller.

\section{CONCLUSIONS}

In this paper, the problem of actuator saturation control of a benchmark structure with active bracing system (ABS) subjected to earthquake excitation using hedgealgebras-based fuzzy controller (HAC) is presented.

It can be emphasized that the hedge-algebras-based fuzzy controller for active control with actuator saturation of the structure are easy in establishment, coherent in implementation, effective in control performance and very efficient in computation time in comparison with the analogical conventional fuzzy controller.

\section{ACKNOWLEDGEMENTS}

This research is funded by Vietnam National Foundation for Science and Technology Development (NAFOSTED) under grant number “107.02-2013.03”. 


\section{REFERENCES}

[1] N. D. Anh, H. L. Bui, N. L. Vu, and D. T. Tran. Application of hedge algebra-based fuzzy controller to active control of a structure against earthquake. Structural Control and Health Monitoring, 20, (4), (2013), pp. 483-495.

[2] S. Pourzeynali, H. H. Lavasani, and A. H. Modarayi. Active control of high rise building structures using fuzzy logic and genetic algorithms. Engineering Structures, 29, (3), (2007), pp. 346-357.

[3] R. Guclu and H. Yazici. Vibration control of a structure with ATMD against earthquake using fuzzy logic controllers. Journal of Sound and Vibration, 318, (1), (2008), pp. 36-49.

[4] M. Marinaki, Y. Marinakis, and G. E. Stavroulakis. Fuzzy control optimized by PSO for vibration suppression of beams. Control Engineering Practice, 18, (6), (2010), pp. 618-629.

[5] Y. F. Wang, D. H. Wang, and T. Y. Chai. Active control of friction-induced self-excited vibration using adaptive fuzzy systems. Journal of Sound and Vibration, 330, (17), (2011), pp. 42014210.

[6] D. Das, T. K. Datta, and A. Madan. Semiactive fuzzy control of the seismic response of building frames with MR dampers. Earthquake Engineering \& Structural Dynamics, 41, (1), (2012), pp. $99-118$.

[7] N. C. Ho, V. N. Lan, and L. X. Viet. Optimal hedge-algebras-based controller: Design and application. Fuzzy Sets and Systems, 159, (8), (2008), pp. 968-989.

[8] N. C. Ho and W. Wechler. Hedge algebras: An algebraic approach to structure of sets of linguistic truth values. Fuzzy Sets and Systems, 35, (3), (1990), pp. 281-293.

[9] N. C. Ho and W. Wechler. Extended hedge algebras and their application to fuzzy logic. Fuzzy Sets and Systems, 52, (3), (1992), pp. 259-281.

[10] N. C. Ho and V. L. Nguyen. Fuzziness measure on complete hedge algebras and quantifying semantics of terms in linear hedge algebras. Fuzzy Sets and Systems, 158, (4), (2007), pp. 452471.

[11] M. E. Uz and M. N. S. Hadi. Optimal design of semi active control for adjacent buildings connected by MR damper based on integrated fuzzy logic and multi-objective genetic algorithm. Engineering Structures, 69, (2014), pp. 135-148.

[12] C. H. Nguyen, W. Pedrycz, T. L. Duong, and T. S. Tran. A genetic design of linguistic terms for fuzzy rule based classifiers. International Journal of Approximate Reasoning, 54, (1), (2013), pp. 1-21.

[13] C. H. Nguyen, V. N. Huynh, and W. Pedrycz. A construction of sound semantic linguistic scales using 4-tuple representation of term semantics. International Journal of Approximate Reasoning, 55, (3), (2014), pp. 763-786.

[14] C. H. Nguyen, T. S. Tran, and D. P. Pham. Modeling of a semantics core of linguistic terms based on an extension of hedge algebra semantics and its application. Knowledge-Based Systems, 67, (2014), pp. 244-262.

[15] D. Vukadinović, M. Bašić, C. H. Nguyen, N. L. Vu, and T. D. Nguyen. Hedge-algebrabased voltage controller for a self-excited induction generator. Control Engineering Practice, 30, (2014), pp. 78-90.

[16] H.-L. Bui, D.-T. Tran, and N.-L. Vu. Optimal fuzzy control of an inverted pendulum. Journal of vibration and control, 18, (14), (2012), pp. 2097-2110.

[17] N. D. Duc, N. L. Vu, D. T. Tran, and H. L. Bui. A study on the application of hedge algebras to active fuzzy control of a seism-excited structure. Journal of Vibration and Control, 18, (14), (2012), pp. 2186-2200. 
[18] H. L. Bui. Active control of a three-story building using hedge-algebras-based fuzzy controller. Vietnam Journal of Mechanics, 33, (2), (2011), pp. 79-94.

[19] H. L. Bui, C. H. Nguyen, N. L. Vu, and C. H. Nguyen. General design method of hedgealgebras-based fuzzy controllers and an application for structural active control. Applied Intelligence, (2015). doi: 10.1007/s10489-014-0638-6.

[20] H. Du, N. Zhang, and F. Naghdy. Actuator saturation control of uncertain structures with input time delay. Journal of Sound and Vibration, 330, (18), (2011), pp. 4399-4412.

[21] C. W. Lim, Y. J. Park, and S. J. Moon. Robust saturation controller for linear time-invariant system with structured real parameter uncertainties. Journal of Sound and Vibration, 294, (1), (2006), pp. 1-14.

\section{APPENDIX 1}

In this section, the idea and basic formulas of HAs theory are summarized based on definitions, theorems and propositions in [1,8-18].

By the term meanings, these could be observed that extremely small $<$ very small $<$ small $<$ approximately small $<$ little small $<$ big $<$ very big $<$ extremely big... So, a following viewpoint is made: term-domains can be modelled by a poset (partially ordered set), a semantics-based order structure. Next, the way to find out this structure will be explained.

Consider DISPLACEMENT as a linguistic variable and let $X$ be its term-set. Assume that its linguistic hedges used to express the DISPLACEMENT are Extremely, Very, Approximately, Little, which for short are denoted by, respectively, $E, V, A$ and $L$, and its primary terms are Positive and Negative, which are denoted by $P_{0}$ and $N e$, respectively. Then, $X=\{P o, V P o, E P o, E A P o, A P o, L A P o, L P o, L N e, N e, A N e, V N e, E N e \ldots\} \cup$ $\{\boldsymbol{0}, \boldsymbol{W}, \mathbf{1}\}$ is a term-domain of DISPLACEMENT, where $\boldsymbol{0}, \boldsymbol{W}$ and $\mathbf{1}$ are specific constants called absolutely Negative, neutral and absolutely Positive, respectively.

A term-domain $X$ can be ordered based on the following observation:

- Each primary term has a sign which expresses a semantic tendency. For instance, Positive has a tendency of "going up", called positive one, and it is denoted by $c^{+}$, while Negative has a tendency of "going down", called negative one, denoted by $c^{-}$. In general, the formula $c^{+} \geq c^{-}$is evident, semantically.

- Each hedge also has a sign. It is positive if it increases the semantic tendency of the primary terms and negative, if it decreases this tendency. For instance, $V$ is positive with respect to both primary terms, while $L$ has a reverse effect and hence it is negative. Denote by $\mathrm{H}^{-}$the set of all negative hedges and by $\mathrm{H}^{+}$the set of all positive ones of DISPLACEMENT.

The term-set $X$ can be considered as an abstract algebra $A X=(X, G, C, H, \leq)$, where $G=\left\{c^{-}, c^{+}\right\}, C=\{\boldsymbol{0}, \boldsymbol{W}, \mathbf{1}\}, H=H^{+} \cup H^{-}$and $\leq$is a partially ordering relation on $X$. It is assumed that $H^{-}=\left\{h_{-1}, \ldots, h_{-q}\right\}$, where $h_{-1}<h_{-2}<\ldots<h_{-q}, H^{+}=\left\{h_{1}\right.$, $\left.\ldots, h_{p}\right\}$, where $h_{1}<h_{2}<\ldots<h_{p}$.

The fuzziness measure of vague terms and hedges of term-domains is defined as follow a $f m: X \rightarrow[0,1]$ is said to be a fuzziness measure of terms in $X$ if:

$$
f m\left(c^{-}\right)+f m\left(c^{+}\right)=1 \text { and } \sum_{h \in H} f m(h u)=f m(u), \quad \text { for } \forall u \in X,
$$

For the constants $\mathbf{0}, \boldsymbol{W}$ and $\mathbf{1}, f m(\boldsymbol{0})=f m(\boldsymbol{W})=f m(\mathbf{1})=0$, 
For $\forall x, y \in X, \forall h \in H, \frac{f m(h x)}{f m(x)}=\frac{f m(h y)}{f m(y)}$.

This proportion does not depend on specific elements, called the fuzziness measure of the hedge $h$ and denoted by $\mu(h)$.

For each fuzziness measure $f m$ on $X$, then:

$$
\begin{gathered}
f m(h x)=\mu(h) f m(x), \quad \text { for every } x \in X, \\
f m\left(c^{-}\right)+f m\left(c^{+}\right)=1, \\
\sum_{i=-q, i \neq 0}^{p} f m\left(h_{i} c\right)=f m(c), c \in\left\{c^{-}, c^{+}\right\}, \\
\sum_{i=-q, i \neq 0}^{p} f m\left(h_{i} x\right)=f m(x), \\
\sum_{i=-q}^{-1} \mu\left(h_{i}\right)=\alpha \text { and } \sum_{i=1}^{p} \mu\left(h_{i}\right)=\beta \text { where } \alpha, \beta>0 \text { and } \alpha+\beta=1 .
\end{gathered}
$$

A function Sign: $X \rightarrow\{-1,0,1\}$ is a mapping which is defined recursively as follows, for $h, h^{\prime} \in H$ and $c \in\left\{c^{-}, c^{+}\right\}$:

$$
\operatorname{Sign}\left(c^{-}\right)=-1, \operatorname{Sign}\left(c^{+}\right)=+1,
$$

$\operatorname{Sign}(h c)=-\operatorname{Sign}(c)$, if $h$ is negative w.r.t. $c$,

$\operatorname{Sign}(h c)=+\operatorname{Sign}(c)$, if $h$ is positive w.r.t. $c$,

$\operatorname{Sign}\left(h^{\prime} h x\right)=-\operatorname{Sign}(h x)$, if $h^{\prime} h x \neq h x$ and $h^{\prime}$ is negative w.r.t. $h$,

$$
\operatorname{Sign}\left(h^{\prime} h x\right)=+\operatorname{Sign}(h x) \text {, if } h^{\prime} h x \neq h x \text { and } h^{\prime} \text { is positive w.r.t. } h \text {, }
$$

$$
\operatorname{Sign}\left(h^{\prime} h x\right)=0, \text { if } h^{\prime} h x=h x \text {. }
$$

Let $f m$ be a fuzziness measure on $X$. A semantically quantifying mapping (SQM) $\varphi: X \rightarrow[0,1]$, which is induced by $f m$ on $X$, is defined as follows:

$$
\begin{aligned}
& \varphi(\boldsymbol{W})=\theta=f m\left(c^{-}\right), \varphi\left(c^{-}\right)=\theta-\alpha f m\left(c^{-}\right)=\beta f m\left(c^{-}\right), \varphi\left(c^{+}\right)=\theta+\alpha f m\left(c^{+}\right), \\
& \varphi\left(h_{j} x\right)=\varphi(x)+\operatorname{Sign}\left(h_{j} x\right)\left\{\sum_{i=\operatorname{Sign}(j)}^{j} f m\left(h_{i} x\right)-\omega\left(h_{j} x\right) f m\left(h_{j} x\right)\right\},
\end{aligned}
$$

where

$$
\begin{aligned}
& j \in\{j:-q \leq j \leq p \& j \neq 0\}=[-q p], \\
& \omega\left(h_{j} x\right)=\frac{1}{2}\left[1+\operatorname{Sign}\left(h_{j} x\right) \operatorname{Sign}\left(h_{p} h_{j} x\right)(\beta-\alpha)\right] .
\end{aligned}
$$

It can be seen that the mapping $\varphi$ is completely defined by $(p+q)$ free parameters: one parameter of the fuzziness measure of a primary term, and $(p+q-1)$ parameters of the fuzziness measure of hedges. 


\section{APPENDIX 2}

In this section, designing of the conventional fuzzy controller (FC) which is similar to HAC is presented.

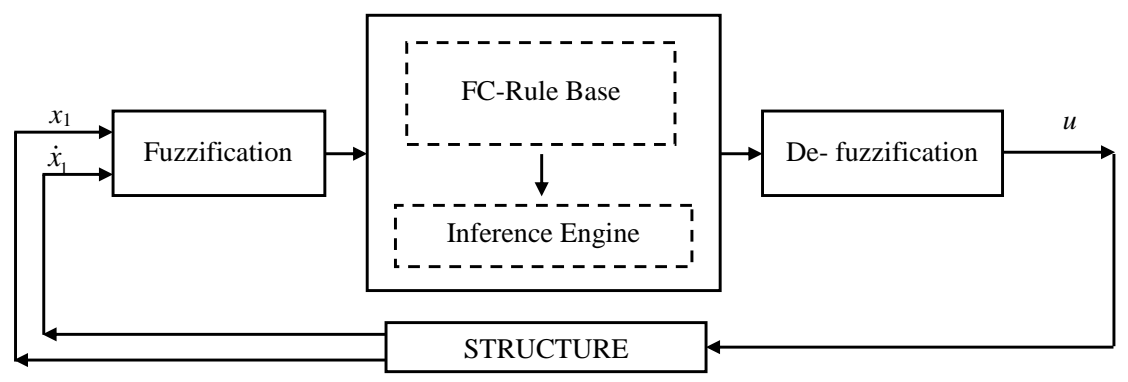

Fig. 18. Operation principle of the analogical FC

Table 5. Rule base of FC

\begin{tabular}{|l|l|l|l|}
\hline$x_{1} \dot{x}_{1}$ & $L N e$ & $Z$ & $L P o$ \\
\hline$N e$ & $V N e$ & $N e$ & $L N e$ \\
\hline$L N e$ & $N e$ & $L N e$ & $Z$ \\
\hline$Z$ & $L N e$ & $Z$ & $L P o$ \\
\hline$L P o$ & $Z$ & $L P o$ & $P o$ \\
\hline$P o$ & $L P o$ & $P o$ & $V P o$ \\
\hline
\end{tabular}
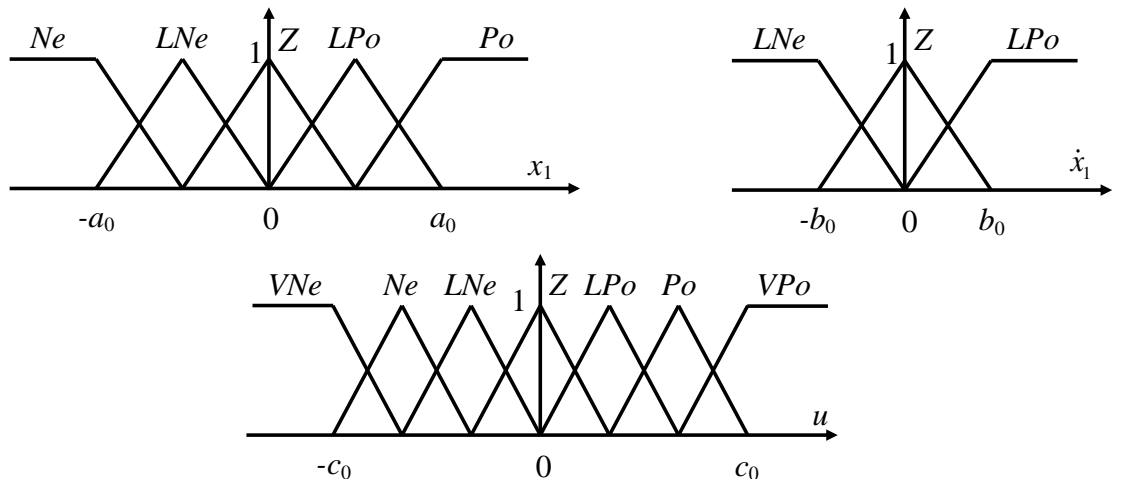

Fig. 19. Fuzzifications of $x_{1}, \dot{x}_{1}$ and $u$

Operation principle of the analogical FC with two-input state variables $x_{1}$ and $\dot{x}_{1}$ and one-output control variable $u$ with actuator saturation is shown in Fig. 18. The rule base of FC is presented in Tab. 5 and fuzzifications of the linguistic variables are shown in Fig. 19, where $Z$ is stood for linguistic value "Zero". Mamdani method and centre gravity method are used as inference engine and de-fuzzification method of FC. 